\title{
Arbitrage and state price deflators in a general intertemporal framework
}

\author{
Elyès JOUINI* Clotilde NAPP ${ }^{\dagger}$ \\ Walter SCHACHERMAYER ${ }^{\ddagger}$
}

June 5, 2007

\begin{abstract}
In securities markets, the characterization of the absence of arbitrage by the existence of state price deflators is generally obtained through the use of the Kreps-Yan theorem.

This paper deals with the validity of this theorem (see Kreps, 1981, and Yan, 1980) in a general framework. More precisely, we say that the KrepsYan theorem is valid for a locally convex topological space $(X, \tau)$, endowed with an order structure, if for each closed convex cone $C$ in $X$ such that $C \supseteq X_{-}$and $C \cap X_{+}=\{0\}$, there exists a strictly positive continuous linear functional on $X$, whose restriction to $C$ is non-positive.

We first show that the Kreps-Yan theorem is not valid for spaces $L^{p}(\Omega, \mathcal{F}, \mathbf{P})$ if $(\Omega, \mathcal{F}, \mathbf{P})$ fails to be sigma-finite.

Then we prove that the Kreps-Yan theorem is valid for topological vector spaces in separating duality $\langle X, Y\rangle$, provided $Y$ satisfies both a "completeness condition" and a "Lindelöf-like condition".

We apply this result to the characterization of the no-arbitrage assumption in a general intertemporal framework.
\end{abstract}

${ }^{*}$ CEREMADE, Université Paris-Dauphine and Institut universitaire de France

${ }^{\dagger}$ CEREMADE, Université Paris-Dauphine and CREST

${ }^{\ddagger}$ TU Wien and Université Paris-Dauphine 


\section{Introduction}

In the applications of stochastic calculus to Mathematical Finance, the following question has turned out to be of particular importance:

$(\mathrm{Q})$ : Let $(X, \tau)$ denote a locally convex topological vector space, endowed with an order structure, and let $Y_{+}$denote the set of nonnegative continuous linear functionals on $X$ i.e. such that $\langle x, y\rangle \geq 0$, for all $x \in X_{+}$. Let $C$ be a closed convex cone in $X$ such that $C \supseteq X_{-}$. If $C \cap X_{+}=\{0\}$, can we find a strictly positive linear functional $y \in Y_{++}$, such that $\left.y\right|_{C} \leq 0$ ? By $y$ "strictly positive", which we denote by $y \in Y_{++}$, we mean that, for each $x \in X_{+}, x \neq 0$, we have $\langle y, x\rangle>0$.

The reason for the relevance of this question in the context of Mathematical Finance is that the condition $C \cap X_{+}=\{0\}$ is related to a no-arbitrage condition, while the existence of a strictly positive functional is related to the existence of an equivalent martingale measure.

Yan (1980) provides a positive answer to the above question if $X$ equals $L^{1}(\Omega, \mathcal{F}, \mathbf{P})$ endowed with its natural order structure over a probability space $(\Omega, \mathcal{F}, \mathbf{P})$ and $\tau$ is given by the norm topology. It is easy to verify that we may replace - mutatis mutandis - the assumption that $(\Omega, \mathcal{F}, \mathbf{P})$ is a probability space by the assumption that $(\Omega, \mathcal{F}, \mathbf{P})$ is a sigma-finite measure space and the space $L^{1}(\Omega, \mathcal{F}, \mathbf{P})$ by any space $L^{p}(\Omega, \mathcal{F}, \mathbf{P})$ for $1 \leq p<\infty$. Kreps (1981) provides a positive answer to the same question for a space $X$ endowed with a Hausdorff topology $\tau$ if either "every subset of $Y_{+}$is weak* separable", or "there exists a countable subset $\left\{x_{1}, \ldots\right\}$ of $X_{+}$such that for every $x \in X_{+}$, there exists $\lambda>0$ and an $n$ such that $x \geq \lambda x_{n}$ " and either " $(X, \tau)$ is normable" or "for every countable subset $\left\{y_{1}, \ldots\right\}$ of $Y_{+}$, there exist real numbers $\lambda_{1}, \ldots$ such that $\lambda_{n}>0$ for all $n, \sum_{n=1}^{\infty} \lambda_{n}=1$ and $\sum_{n=1}^{\infty} \lambda_{n} y_{n} \in Y_{+}$". In Yan (1980) as in Kreps (1981), the first part of the proof consists in a Hahn-Banach separation argument. The second part of the proof relies on exhaustion arguments for Yan and separability arguments for Kreps (see Schachermayer, 1994, for a presentation of the proof).

In the next we shall say that the Kreps-Yan theorem is valid for an ordered topological vector space $\left(X, \tau, X_{+}\right)$(simply denoted by $(X, \tau)$ if the order structure is obvious) if an affirmative answer can be given to the above question (Q). This paper deals with the validity of the Kreps-Yan theorem for specific spaces $(X, \tau)$.

It is shown in Jouini-Napp (2001) that if a closed convex cone $C$ in some

specific space $L^{1}(\widehat{\Omega}, \widehat{\mathcal{F}}, \widehat{\mu})$, where $(\widehat{\Omega}, \widehat{\mathcal{F}}, \widehat{\mu})$ is a not sigma-finite measure space, 
satisfies the assumptions of the Kreps-Yan theorem, i.e. $C \supseteq L_{-}^{1}(\widehat{\Omega}, \widehat{\mathcal{F}}, \widehat{\mu})$ and $C \cap L_{+}^{1}(\widehat{\Omega}, \widehat{\mathcal{F}}, \widehat{\mu})=\{0\}$, together with an additional technical condition (that enables to rewrite the problem in terms of sigma-finite measure spaces), then we can find a strictly positive $y \in L^{\infty}(\widehat{\Omega}, \widehat{\mathcal{F}}, \widehat{\mu})$ such that $\left.y\right|_{C} \leq 0$. The question investigated in Section 2 is whether this technical condition may be dropped, i.e., whether the Kreps-Yan theorem as stated above also extends to non-sigma-finite measure spaces $(\widehat{\Omega}, \widehat{\mathcal{F}}, \widehat{\mu})$. We show in Section 2 that the answer is negative in general.

Section 3 deals with a general version of the Kreps-Yan theorem in the context of topological vector spaces in duality. Let $\langle X, Y\rangle$ be in separating duality. We consider the topological vector space $(X, \tau)$, where $\tau$ denotes any topology compatible with the duality. We show that the Kreps-Yan theorem is valid for $(X, \tau)$ if 1) for every sequence $\left(y_{n}\right)_{n=1}^{\infty} \in Y$ there are strictly positive numbers $\left(\alpha_{n}\right)_{n=1}^{\infty}$ such that $\sum_{n=1}^{\infty} \alpha_{n} y_{n}$ converges in $Y$ with respect to the $\sigma(Y, X)$-topology ("Completeness" condition) and 2) for every family $\left(y_{\alpha}\right)_{\alpha \in I}$ in $Y_{+}$, there is a countable subset $\left(y_{\alpha_{n}}\right)_{n=1}^{\infty}$ such that for every $x \in X_{+}$for which there is some $\alpha \in I$ with $\left\langle x, y_{\alpha}\right\rangle>0$, we can find some $n \in \mathbb{N}$ such that $\left\langle x, y_{\alpha_{n}}\right\rangle>0$ ("Lindelöf-like condition").

This version of the Kreps-Yan theorem is then applied in Section 4 to some concrete examples. For instance, we consider the above mentioned space $X=$ $L^{1}(\widehat{\Omega}, \widehat{\mathcal{F}}, \widehat{\mu})$ considered in Jouini-Napp (2001), endowed not anymore with the norm topology but with a (weaker) topology $\tau$ compatible with the duality $\langle X, Y\rangle$ for a space $Y$ strictly smaller than $L^{\infty}(\widehat{\Omega}, \widehat{\mathcal{F}}, \widehat{\mu})$ given by the set of equivalence classes of adapted processes $y=\left(y_{t}\right)_{t \in R_{+}}$such that the trajectories $t \mapsto y_{t}(\omega)$ are uniformly bounded and càdlàg. We also consider the space $X=\mathcal{M}\left(\Omega \times R_{+}, \mathcal{O}\right)$ of equivalence classes of finite measures $\mu$ on the optional sigma-algebra $\mathcal{O}$ endowed with a topology $\tau$ compatible with the duality $\langle X, Y\rangle$ for the same space $Y$. We show that in both cases (as well as for some variants of them) the Kreps-Yan theorem is valid for $(X, \tau)$.

Finally, in Section 5, we give applications to Mathematical Finance. We characterize the assumption of no free lunch (a slightly stronger assumption than the one of no-arbitrage) in a general model of investment.

Results on the Kreps-Yan theorem and the characterization of the no-arbitrage condition have been obtained in (among others) Duffie-Huang (1986), Stricker (1990), Delbaen (1992), Clark (1993), Lakner (1993), Frittelli-Lakner (1994), Schachermayer (1994), Delbaen-Schachermayer (1994, 1998), Klein-Schachermayer (1996). 
In Duffie-Huang (1986), the space $(X, \tau)$ is normed and separable. The application of Yan's theorem to the characterization of the no-arbitrage condition is introduced in Stricker $(1990)$; there the space $(X, \tau)$ is the space $L^{p}(\Omega, \mathcal{F}, \mathbf{P})$ over a probability space $(\Omega, \mathcal{F}, \mathbf{P})$ for $p \in[1, \infty)$, endowed with the norm topology. Delbaen (1992) deals with the space $L^{\infty}(\Omega, \mathcal{F}, \mathbf{P})$ over a probability space $(\Omega, \mathcal{F}, \mathbf{P})$, endowed with the Mackey topology $\tau\left(L^{\infty}, L^{1}\right)$. In Clark (1993), a separable Banach space $X$ is considered; it is noted that one may use the classical Halmos-Savage theorem (see Halmos-Savage, 1949) to formalize the exhaustion argument in Yan (1980). In Schachermayer (1994), the space $(X, \tau)$ is the space $L^{\infty}(\Omega, \mathcal{F}, \mathbf{P})$ for a probability space $(\Omega, \mathcal{F}, \mathbf{P})$, endowed with the weak* topology $\sigma\left(L^{\infty}, L^{1}\right)$. Lakner (1993) deals with topological spaces in (not necessarily separating) duality $\langle M, N\rangle$, where $M$ is a subspace of $L^{1}(\Omega, \mathcal{F}, \mathbf{P})$ and $N$ a subspace of $L^{\infty}(\Omega, \mathcal{F}, \mathbf{P})$ for a probability space $(\Omega, \mathcal{F}, \mathbf{P})$. Delbaen-Schachermayer (1994, $1998)$ consider the space $L^{\infty}(\Omega, \mathcal{F}, \mathbf{P})$ over a probability space $(\Omega, \mathcal{F}, \mathbf{P})$, endowed with the norm topology.

In Klein-Schachermayer (1996b), a quantitative version of the Halmos-Savage theorem is obtained and applied to characterize the absence of asymptotic arbitrage (compare Kabanov-Kramkov $(1994,1998)$ and Klein-Schachermayer (1996a)).

\section{Yan's theorem does not extend to the non-sigma-finite case}

It is shown in Jouini-Napp (2001) that if a closed convex cone $C$ in some space $L^{1}(\widehat{\Omega}, \widehat{\mathcal{F}}, \widehat{\mu})$, where $(\widehat{\Omega}, \widehat{\mathcal{F}}, \widehat{\mu})$ is a (not sigma-finite) measure space, satisfies the conditions of the Kreps-Yan theorem, i.e. $C \supseteq L_{-}^{1}(\widehat{\Omega}, \widehat{\mathcal{F}}, \widehat{\mu})$ and $C \cap L_{+}^{1}(\widehat{\Omega}, \widehat{\mathcal{F}}, \widehat{\mu})=$ $\{0\}$, and an additional condition (to be described below), then we can find a strictly positive continuous linear functional on $X$ whose restriction to $C$ is nonpositive. The additional condition, although having an intuitive economic interpretation, is mathematically not very satisfactory. The question investigated in this section is whether the Kreps-Yan theorem is valid for non-sigma-finite measure spaces $(\widehat{\Omega}, \widehat{\mathcal{F}}, \widehat{\mu})$. In other words, can the additional condition in Jouini-Napp (2001) be dropped?

The framework of Jouini-Napp (2001) is essentially the following. Fix a filtered probability space $\left(\Omega, \mathcal{F},\left(\mathcal{F}_{t}\right)_{t \in R_{+}}, \mathbf{P}\right)$ satisfying the usual conditions of saturatedness and right continuity and define the (non-sigma-finite) measure space $(\widehat{\Omega}, \widehat{\mathcal{F}}, \widehat{\mu})$ as the direct sum of the probability spaces $\left(\Omega, \mathcal{F}_{t}, \mathbf{P}\right)$, i.e. $\widehat{\Omega}$ is the disjoint union 
of continuum many copies $\left(\Omega_{t}\right)_{t \in R_{+}}$of $\Omega, \widehat{\mathcal{F}}$ is the sigma-algebra of sets $\hat{A} \subseteq \widehat{\Omega}$ such that $\hat{A} \cap \Omega_{t} \in \mathcal{F}_{t}$, for each $t \in R_{+}$, and $\widehat{\mu}$ induces on each $\left(\Omega_{t},\left.\widehat{\mathcal{F}}\right|_{\Omega_{t}}\right)$ the original probability measure $\mathbf{P}$.

We then may represent the Banach lattice $X=L^{1}(\widehat{\Omega}, \widehat{\mathcal{F}}, \widehat{\mu})$ as the space of all families $f=\left(f_{t}\right)_{t \in R_{+}}$such that for all $t \in R_{+}, f_{t} \in L^{1}\left(\Omega, \mathcal{F}_{t}, \mathbf{P}\right)$ and

$$
\|f\|_{L^{1}(\widehat{\Omega}, \widehat{\mathcal{F}}, \widehat{\mu})}=\sum_{t \in R_{+}}\left\|f_{t}\right\|_{L^{1}\left(\Omega, \mathcal{F}_{t}, \mathbf{P}\right)}<\infty .
$$

The finiteness of the above sum implies in particular that $f_{t}=0$ for all but countably many $t$ 's in $R_{+}$. The dual space of $X$ may be represented as $L^{\infty}(\widehat{\Omega}, \widehat{\mathcal{F}}, \widehat{\mu})$, which is defined as the space of all families $g=\left(g_{t}\right)_{t \in R_{+}}$such that, for all $t \in R_{+}$, $g_{t} \in L^{\infty}\left(\Omega, \mathcal{F}_{t}, \mathbf{P}\right)$ and

$$
\|g\|_{L^{\infty}(\widehat{\Omega}, \widehat{\mathcal{F}}, \widehat{\mu})}=\sup _{t \in R_{+}}\left\|g_{t}\right\|_{L^{\infty}\left(\Omega, \mathcal{F}_{t}, \mathbf{P}\right)}<\infty .
$$

The scalar product is defined by $\langle f, g\rangle=\sum_{t \in R_{+}}\left\langle f_{t}, g_{t}\right\rangle$.

The additional condition introduced in Jouini-Napp (2001) consists in assuming that there exists a sequence $d=\left(d_{n}\right)_{n \in \mathbb{N}}$ in $R_{+}$such that for each time $t^{*} \geq 0$, for all $B_{t^{*}}$ in $F_{t^{*}}$ of positive probability, there exists $c$ in $C$ of the form $c_{t^{*}}=0$ outside $B_{t^{*}}, c_{t}=0$ for all $t<t^{*}, c_{t} \geq 0$ for all $t>t^{*}$, and there exists $d_{n} \in d$, $P\left[c_{d_{n}}>0\right]>0$ (see Jouini-Napp, 2001, for an economic interpretation of this condition). It is shown that if a closed convex cone $C$ in $L^{1}(\widehat{\Omega}, \widehat{\mathcal{F}}, \widehat{\mu})$ satisfies $C \supseteq L_{-}^{1}(\widehat{\Omega}, \widehat{\mathcal{F}}, \widehat{\mu}), C \cap L_{+}^{1}(\widehat{\Omega}, \widehat{\mathcal{F}}, \widehat{\mu})=\{0\}$ and this additional condition, then there exists $g=\left(g_{t}\right)_{t \in R_{+}} \in L^{\infty}(\widehat{\Omega}, \widehat{\mathcal{F}}, \widehat{\mu})$ with $P\left[g_{t}>0\right]=1$ for each $t \in R_{+}$, such that $C$ is contained in the half-space $H_{g}=\left\{f \in L^{1}(\widehat{\Omega}, \widehat{\mathcal{F}}, \widehat{\mu}):\langle f, g\rangle \leq 0\right\}$. We now shall provide a simple example showing that the additional condition cannot be dropped, i.e. that the Kreps-Yan theorem does not extend to non-sigma-finite measure spaces (in general).

Example 2.1. Let $\Omega$ simply consist of one point, so that $\left(\Omega, \mathcal{F}_{t}, \mathbf{P}\right)$ is the trivial probability space, for each $t \in R_{+}$. In this case, $\left.L^{1}(\widehat{\Omega}, \widehat{\mathcal{F}}, \widehat{\mu})\right)$ may be identified with $l^{1}\left(R_{+}\right)=\left\{\left(f_{t}\right)_{t \in R_{+}}, f_{t} \in R, \sum_{t \in R_{+}}\left|f_{t}\right|<\infty\right\}$ and $L^{\infty}(\widehat{\Omega}, \widehat{\mathcal{F}}, \widehat{\mu})$ with $l^{\infty}\left(R_{+}\right)$. Fix $t_{0} \in R_{+}$and define $C \subseteq l^{1}\left(R_{+}\right)$by

$$
C=\left\{f=\left(f_{t}\right)_{t \in R_{+}}: f_{t_{0}} \leq-\sup _{t \in R_{+}} f_{t}\right\} .
$$


Noting that for every $f \in l_{+}^{1}\left(R_{+}\right), f \neq 0$, we have $\sup _{t \in R_{+}} f_{t}>0$, we see that $C \cap l_{+}^{1}\left(R_{+}\right)=\{0\}$. It is also straightforward to verify that $C$ is closed in $l^{1}\left(R_{+}\right)$ and that $C$ contains the negative orthant $l_{-}^{1}\left(R_{+}\right)$.

We shall now show that, for each $g=\left(g_{t}\right)_{t \in R_{+}}$in $l^{\infty}\left(R_{+}\right)$such that $g_{t}>0$ for all $t \in R_{+}, C$ is not contained in the half space $H_{g}=\left\{f \in l^{1}\left(R_{+}\right):\langle f, g\rangle \leq 0\right\}$. Indeed, for such an element $g$ we can find $\varepsilon>0$ such that $g_{t}>\varepsilon$ for infinitely many $t^{\prime}$ 's. Let $M>g_{t_{0}} / \varepsilon$ and find $t_{1}, \ldots, t_{M}$ such that $g_{t_{i}} \geq \varepsilon$, for $i \in\{1, \ldots, M\}$. The element

$$
f=-e_{t_{0}}+\sum_{i=1}^{M} e_{t_{i}}
$$

$e_{t}$ denoting the $t^{\text {th }}$ unit vector of $l^{1}\left(R_{+}\right)$, is an element of $C$ for which we have

$$
\langle f, g\rangle=-g_{t_{0}}+\sum_{i=1}^{M} g_{t_{i}}>0 .
$$

i.e. $f$ is not in the half space $H_{g}$.

Another way to remedy the phenomenon encountered in the above example - i.e., the failure of the Kreps Yan theorem - is to weaken the topology on the space $L^{1}(\widehat{\Omega}, \widehat{\mathcal{F}}, \widehat{\mu})$. In fact, this will be the main topic of the remainder of this paper.

To motivate this idea recall that in the above example we have considered the norm topology on $L^{1}(\widehat{\Omega}, \widehat{\mathcal{F}}, \widehat{\mu})$ so that its dual equals $L^{\infty}(\widehat{\Omega}, \widehat{\mathcal{F}}, \widehat{\mu})$. Considering the elements $g=\left(g_{t}\right)_{t \in R_{+}} \in L^{\infty}(\widehat{\Omega}, \widehat{\mathcal{F}}, \widehat{\mu})$ as functions on $\Omega \times R_{+}$note that, for fixed $\omega \in \Omega$, the function $t \mapsto g_{t}(\omega)$ does not obey any continuity or measurability requirements.

In any case, the space $Y=L^{\infty}(\widehat{\Omega}, \widehat{\mathcal{F}}, \widehat{\mu})$ seems much too big for a useful economic interpretation and should be replaced by a space $Y$ of more regular processes, e.g., the adapted bounded processes $\left(y_{t}\right)_{t \in R_{+}}$which almost surely have càdlàg (or càglàd, or continuous) trajectories.

This leads us to consider the space $X=L^{1}(\widehat{\Omega}, \widehat{\mathcal{F}}, \widehat{\mu})$ in duality with the spaces $Y$ proposed above and to equip $X$ with a topology $\tau$ compatible with the dual pair $\langle X, Y\rangle$, We shall see below that in this setting we do have a positive result of Kreps-Yan type.

Why does the above counterexample not carry over to this setting? Observe, for example, that, using the above notation, $e_{t_{n}} \in X$ converges to $e_{t} \in X$ with 
respect to the topologies $\tau$ proposed above, if $t_{n}$ tends to $t$ (from above in the case of càdlàg, from below in the case of càglàd and in an arbitrary manner in the case of continuous trajectories). This convergence makes also good sense economically. Finally observe that in Example 2.1, the closure of the cone $C$ with respect to the above topologies does intersect the positive cone of $X=L^{1}(\widehat{\Omega}, \widehat{\mathcal{F}}, \widehat{\mu})$ in a non-trivial way (in fact the closure of $C$ equals the entire space $X=L^{1}(\widehat{\Omega}, \widehat{\mathcal{F}}, \widehat{\mu})$ ).

In the next section we develop a framework in the context of topological vector spaces in duality to prove a general version of the Kreps-Yan theorem which then is applied in Section 4 to the above sketched situation as well as to some variants of it. Finally in Section 5 we give applications to Mathematical Finance.

\section{A general version of the Kreps-Yan theorem for ordered locally convex spaces}

We consider a pair $\langle X, Y\rangle$ of vector spaces in separating duality. Let $(X, \tau)$ be a vector space, endowed with a topology compatible with the duality ${ }^{1}$. On $X$ we are given a cone $X_{+}$, of non-negative elements, such that $X_{+} \cap\left(-X_{+}\right)=\{0\}$ and $X_{+}-X_{+}=X$. Letting $x_{1} \leq x_{2}$ if $x_{2}-x_{1} \in X_{+}$we have endowed $X$ with an order structure. Let $Y_{+}=\left\{y \in Y:\langle x, y\rangle \geq 0\right.$, for $\left.x \in X_{+}\right\}$and $Y_{++}=\left\{y \in Y:\langle x, y\rangle>0\right.$, for $\left.x \in X_{+}, x \neq 0\right\}$.

We assume from now on that $(X, \tau)$ satisfies the following mild completeness assumption.

Assumption (C): For every sequence $\left(y_{n}\right)_{n=1}^{\infty} \in Y$ there are strictly positive numbers $\left(\alpha_{n}\right)_{n=1}^{\infty}$ such that $\sum_{n=1}^{\infty} \alpha_{n} y_{n}$ converges in $Y$ with respect to the $\sigma(Y, X)$ topology.

We note that this is an assumption on the locally convex topological vector space $X$, as $(X, \tau)$ determines $Y$ as well as the $\sigma(Y, X)$-topology on $Y$.

Note, for example, that if there exists a norm $\|\cdot\|_{Y}$ on $Y$, under which $Y$ is a Banach space and which induces a finer topology than $\sigma(Y, X)$, assumption (C) is satisfied. This observation will take care of all the examples considered in the next section.

For a (rather pathological) example where the failure of assumption (C) causes the Kreps-Yan theorem not to hold true we refer to Schachermayer (2000).

\footnotetext{
${ }^{1}$ We recall that a topology on $X$ is said to be compatible with the duality $\langle X, Y\rangle$ if it is locally convex, and if the dual of $X$ for this topology is identical to $Y$.
} 
The next assumption is more restrictive in applications as it rules out economically more important situations, e.g., the case $(X, \tau)=\left(L^{1}(\widehat{\Omega}, \widehat{\mathcal{F}}, \widehat{\mu}),\|\cdot\|_{1}\right)$ considered in the previous section. While the letter $(\mathrm{C})$ above stands for "completeness" the notation (L) alludes to the "Lindelöf condition" encountered in general topology.

Assumption (L): For every family $\left(y_{\alpha}\right)_{\alpha \in I}$ in $Y_{+}$, there is a countable subset $\left(y_{\alpha_{n}}\right)_{n \in \mathbb{N}}$ such that for every $x \in X_{+}$for which there is some $\alpha \in I$ with $\left\langle x, y_{\alpha}\right\rangle>0$, we can find some $n \in \mathbb{N}$ such that $\left\langle x, y_{\alpha_{n}}\right\rangle>0$.

Now we are ready to state the main theorem of this paper.

Theorem 3.1. If $(X, \tau)$ satisfies $(C)$ and $(L)$, then the Kreps-Yan theorem holds true, i.e., for each closed convex cone $C$ in $X$ such that $C \supseteq X_{-}$and $C \cap X_{+}=\{0\}$, there exists a strictly positive continuous linear functional $y \in Y_{++}$, such that $\left.y\right|_{C} \leq 0$.

Proof Now that we have isolated the crucial features in the above definitions, the proof is almost reduced to a formality. The first step consists of a (HahnBanach type) separation theorem. Note that the space $X$ is locally convex and that $C$ is a nonempty closed convex set in $X$. Fix $x \in X_{+}, x \neq 0$; noting that $x \notin C$, there exists a closed hyperplane strictly separating $C$ and $\{x\}$. Since $\tau$ is compatible with the duality $\langle X, Y\rangle$, any continuous linear functional on $X$ is induced by some $y \in Y$. Hence we obtain that for all $x \neq 0$ in $X_{+}$, there exists $y_{x} \neq 0$ in $Y$ such that for some $\alpha$,

$$
\left\langle c, y_{x}\right\rangle \leq \alpha<\left\langle x, y_{x}\right\rangle \quad \text { for all } c \in C .
$$

Since $C$ is a convex cone, we can take $\alpha=0$. Since $X_{-} \subseteq C,\left\langle k, y_{x}\right\rangle \geq 0$ for all $k$ in $X_{+}$and therefore $y_{x} \in Y_{+}$.

We have so far obtained that the set $\mathcal{G} \equiv\left\{y \in Y_{+},\left.y\right|_{C} \leq 0\right\}$ is not reduced to $\{0\}$. In fact, for each fixed $x \in X_{+}, x \neq 0$, there is $y_{x} \in \mathcal{G}$ satisfying $\left\langle x, y_{x}\right\rangle>0$. We need to prove that $\mathcal{G}$ has a nonempty intersection with $Y_{++}$. To do so we apply an exhaustion argument. We consider the subset $\left(y_{x_{n}}\right)_{n=1}^{\infty}$ of $\left(y_{x}\right)_{x \in X_{+}}$given by condition (L). Condition (C) ensures the existence of strictly positive numbers $\left(\alpha_{n}\right)_{n=1}^{\infty}$ such that $\sum_{n=1}^{\infty} \alpha_{n} y_{n}$ converges for the weak topology $\sigma(Y, X)$ to some $y \in Y$. It is then clear that $y$ belongs to $Y_{++}$and that $\left.y\right|_{C} \leq 0$.

Remark 1. We observe that the assumptions $(C)$ and $(L)$ of the above theorem depend on the topology $\tau$ chosen on $X$ only to the extent that $\tau$ determines the dual space $Y$. In other words, $(C)$ and $(L)$ are satisfied for $(X, \tau)$ if and only if they are satisfied for $(X, \sigma(X, Y))$. 
Notice that (L) is the natural condition which reduces the proof of the KrepsYan theorem to a formality. To further motivate the introduction of condition (L), we recall the following well-known result which is a straightforward consequence of the Halmos-Savage theorem from Mathematical Statistics. We also mention that its importance for Mathematical Finance has been noticed by Clark (for further refinements arising in the context of asymptotic arbitrage we also refer to Kabanov-Kramkov $(1994,1998)$ and Klein-Schachermayer(1996a, 1996b)).

Lemma 3.2. If $(\Omega, \mathcal{F}, \mu)$ is a sigma-finite measure space and $(X, \tau)=\left(L^{p}(\Omega, \mathcal{F}, \mu),\|\cdot\|_{p}\right)$, for $1 \leq p<\infty$, or $(X, \tau)=\left(L^{\infty}(\Omega, \mathcal{F}, \mu), \sigma\left(L^{\infty}, L^{1}\right)\right)$, then assumption $(L)$ is satisfied.

\section{Some concrete examples}

We now consider various situations to which Theorem 3.1 may be applied.

Example 4.1. Let $X=L^{1}(\widehat{\Omega}, \widehat{\mathcal{F}}, \widehat{\mu})$ be as in Section 2 and let $Y$ be the space of equivalence classes of adapted processes $y=\left(y_{t}\right)_{t \in R_{+}}$such that the trajectories $t \mapsto y_{t}(\omega)$ are uniformly bounded and càdlàg, for almost all $\omega \in \Omega$, modulo the processes vanishing outside an evanescent set. This is a Banach space for the obvious norm $\|\cdot\|_{\infty}$ and therefore has property $(C)$. Property $(L)$ is verified by the subsequent Lemma 4.5. The fact that $Y$ separates the points of $X$ and vice versa is not completely trivial but easily verified.

On $X$ we consider any vector space topology $\tau$ which is between the $\sigma(X, Y)$ and the Mackey $(X, Y)$-topology.

Example 4.2.a (resp. b) We now consider $Y$ as above but the word càdlàg replaced by càglàd (resp. continuous). In order to have that $Y$ separates the points of $X$, we have to change the definition of $X$ : instead of requiring $g_{t} \in$ $L^{\infty}\left(\Omega, F_{t}, P\right)$, we have to require that $g_{t} \in L^{\infty}\left(\Omega, F_{t-}, P\right)$.

We now can go one step further: instead of only considering the space $X=$ $L^{1}(\widehat{\Omega}, \widehat{\mathcal{F}}, \widehat{\mu})$ we may enlarge this space to consist of all adapted processes with integrable variation which is a more natural object in the context of stochastic integration theory and still in separating duality with the above proposed spaces $Y$. For an economic interpretation of this larger space we refer to Section 5 below. 
Example 4.3. (variant of Example 4.1) Let $X=\mathcal{M}\left(R_{+} \times \Omega, \mathcal{O}\right)$ be the space of equivalence classes of finite measures $\mu$ on the optional sigma-algebra $\mathcal{O}$, vanishing on the evanescent sets. The variation norm $\|\cdot\|_{1}$ makes this Banach space an abstract $L$-space in which $L^{1}(\widehat{\Omega}, \widehat{\mathcal{F}}, \widehat{\mu})$ is naturally and isometrically embedded (it is the subspace of measures supported by countable subsets of $\left.R_{+}\right)$.

Another way of representing $X$ (by integrating the measure $\mu$ on each $\{\omega\} \times R_{+}$) is as the space of adapted càdlàg processes of integrable variation.

$X$ is in separating duality with the space $Y$ defined in Example 4.1. If we equip $X$ with a topology $\tau$ compatible with the duality $\langle X, Y\rangle$ then again $(L)$ follows from the subsequent Lemma 4.5.

Example 4.4. a (resp. b) (variant of Examples 4.2 a. (resp. b.)) Take $Y$ as in Example 4.2 a (resp. b), and $X$ similarly as in Example 4.3, but now the optional sigma-algebra $\mathcal{O}$ replaced by the predictable sigma-algebra $\mathcal{P}$. In this case $X$ can be represented as the space of adapted càglàd processes of integrable variation.

We now state the crucial result. Recall that we assume throughout the paper that $\left(\Omega, \mathcal{F},\left(\mathcal{F}_{t}\right)_{t \in R_{+}}, \mathbf{P}\right)$ satisfies the usual conditions of right continuity and saturatedness.

Lemma 4.5. In the setting of Examples 4.3, $4.4 \mathrm{a}$ and $4.4 \mathrm{~b}$, let $\left(y_{\alpha}\right)_{\alpha \in I}$ be a family of elements in $Y_{+}$. Then there is a countable subset $\left(y_{\alpha_{n}}\right)_{n \in \mathbb{N}}$ such that for $x \in X_{+},\left\langle x, y_{\alpha_{n}}\right\rangle=0$ for all $n \in \mathbb{N}$, implies that $\left\langle x, y_{\alpha}\right\rangle=0$, for all $\alpha \in I$.

It follows that in all the Examples 4.1-4.4, condition $(L)$ is satisfied.

Proof A) We start with Example 4.4 b., i.e. the case where $Y$ consists of the (equivalence classes) of adapted continuous processes $y=(y(t))_{t \in R_{+}}$.

Let $\left(y_{\alpha}\right)_{\alpha \in I}$ be a given family in $Y_{+}$as in the statement of condition (L). Let $\left(U_{i}\right)_{i=1}^{\infty}$ be a basis for the topology on $R_{+}$, e.g., an enumeration of the open intervals with rational endpoints.

For each $i \in \mathbb{N}$ and $\alpha \in I$ denote by $A_{i, \alpha}$ the set

$$
A_{i, \alpha}=\left\{\omega: y_{\alpha}(t)(\omega)>0, \text { for all } t \in U_{i}\right\}
$$

which clearly is an $\mathcal{F}$-measurable subset of $\Omega$ (strictly speaking, it is an equivalence class modulo $\mathbf{P}$-null sets). 
Now proceed similarly as in the proof of the Halmos-Savage theorem to choose, for each $i \in \mathbb{N}$, a sequence $\left(\alpha_{n}(i)\right)_{n=1}^{\infty}$ such that the $\mathbf{P}$-measure of

$$
A_{i}=\bigcup_{n=1}^{\infty} A_{i, \alpha_{n}(i)}
$$

is maximal.

We claim that the countable family $\left(y_{\alpha_{n}(i)}\right)_{n, i}$ satisfies our requirements. Indeed, form $y=\sum_{n, i} \lambda_{n, i} y_{\alpha_{n}(i)}$, where $\lambda_{n, i}>0$ are chosen such that the sum converges and denote by $S$ its supporting set

$$
S=\{(t, \omega): y(t)(\omega)>0\},
$$

which is predictable and welldefined (modulo evanescent sets). We claim that, for each $\alpha \in I$, the supporting set

$$
S_{\alpha}=\left\{(t, \omega): y_{\alpha}(t)(\omega)>0\right\}
$$

is contained in $S$, up to an evanescent set. Indeed, suppose there is $\bar{\alpha} \in I$ for which this is not the case. Denoting by $S(\omega)$ and $S_{\bar{\alpha}}(\omega)$ the intersections of the sets $S$ and $S_{\bar{\alpha}}$ with $R_{+} \times\{\omega\}$, observe that these sets are (almost surely) open subsets of $R_{+}$. Whence, if $\mathbf{P}\left[\omega: S_{\bar{\alpha}}(\omega) \backslash S(\omega) \neq \emptyset\right]>0$ we may find some $i \in \mathbb{N}$, such that

$$
\mathbf{P}\left[\omega: S_{\bar{\alpha}}(\omega) \supseteq U_{i} \text { and } S(\omega) \supseteq U_{i}\right]>0 .
$$

But this is a contradiction to the choice of $\left(\alpha_{n}(i)\right)_{n=1}^{\infty}$.

Hence any $x \in X_{+}$(which in the setting of Example 4.4 is a positive measure on the predictable sigma-algebra $\mathcal{P}$ ) s.t. $\left\langle x, y_{\alpha_{n}(i)}\right\rangle=0$, for all $n, i$, vanishes on the predictable set $S$ (up to an evanescent set) and therefore satisfies $\left\langle x, y_{\alpha}\right\rangle=0$, for all $\alpha \in I$.

B) We now proceed to the situation of Example 4.3, where $Y$ consists of càdlàg processes $y=(y(t))_{t \in R_{+}}$, which is more delicate.

Given $\left(y_{\alpha}\right)_{\alpha \in I} \in Y_{+}$repeat the above argument to find $\left(y_{\alpha_{n}(i)}\right)_{n, i}, y$ and $S$ as above. But now the set $S$ only is in the optional sigma-algebra as the sets

$$
S(\omega)=\{\omega: y(t)(\omega)>0\}
$$

are not necessarily open anymore (they are the support of a càdlàg function). 
For fixed $\omega \in \Omega$ denote by $L(\omega)$ the countable set of points where $y(t)(\omega)$ vanishes and such that it is positive in an open interval to the right of $t$, i.e.,

$$
\begin{gathered}
L(\omega)=\left\{t \in R_{+}: \text {there is } \varepsilon=\varepsilon(t, \omega)>0\right. \text { such that } \\
y(s)(\omega)>0 \text { for } s \in] t, t+\varepsilon[\text { and } y(t)=0\} .
\end{gathered}
$$

Why is this set important for us? For each $\alpha \in I$ one verifies as above that the interior of the set $S_{\alpha}(\omega)$ is contained in $S(\omega) \mathbf{P}$-almost surely; yet there may be points $t \in R_{+}$such that $y_{\alpha}(t)(\omega)>0$ while $y(t)(\omega)=0$. But a moment's reflection reveals that these points must be contained in $L(\omega)$.

Let $L=\bigcup_{\omega \in \Omega} L(\omega) \times\{\omega\}$. We show below that $L$ may be exhausted by a sequence $\left(T_{k}\right)_{k=1}^{\infty}$ of stopping times, i.e.,

$$
L \subseteq \bigcup_{k=1}^{\infty}\left\{\left(T_{k}(\omega), \omega\right): T_{k}(\omega)<\infty\right\},
$$

up to an evanescent set ${ }^{2}$.

Admitting this for the moment we proceed as follows: first we show that, for each $\alpha \in I, S_{\alpha}$ is contained in $S \cup L$, up to an evanescent set; this works just as in part A) above.

But the set $L$ still needs special treatment: for $\alpha \in I$ and $k \in \mathbb{N}$, let

$$
A_{k, \alpha}=\left\{\omega: T_{k}(\omega)<\infty \text { and } y_{\alpha}\left(T_{k}\right)(\omega)>0\right\}
$$

and apply the previous argument to find sequences $\left(\beta_{n}(k)\right)_{n=1}^{\infty} \in I$ such that the $\mathbf{P}$-measure of

$$
A_{k}=\bigcup_{n=1}^{\infty} A_{k, \beta_{n}(k)}
$$

is maximal. Now one verifies, similarly as in part A), that the countable family $\left(y_{\alpha_{n}(i)}\right)_{n, i} \cup\left(y_{\beta_{n}(k)}\right)_{n, k}$ satisfies the requirements of $(\mathrm{L})$.

To make the proof selfcontained (i.e., not relying on Dellacherie's theorem, mentioned in footnote 2) we still have to construct the stopping times $T_{k}$. Let

$$
\begin{gathered}
T_{0}(\omega)=\inf \{t \geq 0: \text { there is } \varepsilon>0 \text { such that } y(s)(\omega)>0, \\
\text { for } t<s<t+\varepsilon \text { and } y(t)(\omega)=0\}
\end{gathered}
$$

\footnotetext{
${ }^{2}$ Since $L$ is optional and for all $\omega, L(\omega)$ is countable, this is a special case of Dellacherie's Theorem (Dellacherie, (1972), chap. VI) that $L$ is indistinguishable from a reunion of graphs of optional times. For the convenience of the reader we give below a proof of this result in our specific setting.
} 
and inductively, for each countable ordinal $\gamma \geq 1$

$$
\begin{gathered}
T_{\gamma}(\omega)=\inf \left\{t>0: t>T_{\eta}(\omega) \text { for } \eta<\gamma \text { and there is } \varepsilon>0\right. \text { such that } \\
y(s)(\omega)>0, \text { for } t<s<t+\varepsilon \text { and } y(t)(\omega)=0\}
\end{gathered}
$$

where, as customary, we let $T_{\gamma}(\omega)=\infty$ if the corresponding set is empty. Using the usual conditions on the filtered space one verifies inductively that each $T_{\gamma}$ is a stopping time. As the family $\left(T_{\gamma}\right)_{\gamma}$ is strictly increasing as long as $T_{\gamma}<\infty$ there is some countable ordinal $\gamma_{0}$ such that $\mathbf{P}\left[T_{\gamma_{0}}=\infty\right]=1$. Renumbering the countable set $\left(T_{\gamma}\right)_{0 \leq \gamma \leq \gamma_{0}}$ by $\left(T_{k}\right)_{k=1}^{\infty}$ we have constructed a sequence of stopping times s.t., for $\mathbf{P}$ almost all $\omega \in \Omega,\left\{T_{k}(\omega): k \in \mathbb{N}\right\} \supseteq L(\omega)$.

This finishes the proof of part B).

C) The case of càglàd trajectories (Example 4.4 a.): This case is similar to case B) but now the delicate point are the following right limit points $R(\omega)$ of the set $S(\omega)$ (plus the point $t=0$ ):

$$
\begin{aligned}
& R(\omega)=\left\{t \in R_{+}: t=0 \text { or there is } \varepsilon>0\right. \text { such that } \\
&y(s)(\omega)>0, \text { for } s \in] t-\varepsilon, t[\text { and } y(t)(\omega)=0\}
\end{aligned}
$$

By a similar argument the set $R=\bigcup_{\omega \in \Omega} R(\omega) \times\{\omega\}$ can now be exhausted by a sequence of predictable ${ }^{3}$ stopping times $\left(T_{k}\right)_{k=1}^{\infty}$.

Indeed, the set $R$ can be written as the union of the sets

$$
R^{(m)}=\left\{(t, \omega): y_{t}(\omega)=0 \text { and } y_{s}(\omega)>0, \text { for } t-\frac{1}{m} \leq s<t\right\} .
$$

The sets $R^{(m)}$ can be exhausted by a sequence of predictable stopping times defined by $T_{0}^{(m)}=0$ and

$$
T_{i}^{(m)}=\inf \left\{t>T_{i-1}^{(m)}: y_{s}(\omega)>0, \text { for } t-\frac{1}{m} \leq s<t \text { and } y_{s}(\omega)>0\right\} .
$$

(This time we do not need transfinite induction.)

Noting that the set $S$ now is predictable, the proof of B) carries over (mutatis mutandis) to finish the proof of $\mathrm{C}$ ).

Finally observe that property (L) for the case of Examples 4.1, $4.2 \mathrm{a}$ and $4.2 \mathrm{~b}$ now follows as special cases of the above arguments.

\footnotetext{
${ }^{3}$ As in footnote 2, we also refer to Dellacherie (1972, Chap. VI): since $R$ is predictable, and for all $\omega \in \Omega, R(\omega)$ is countable, $R$ is indistinguishable from a reunion of graphs of predictable stopping times.
} 


\section{Applications to Mathematical Finance}

As in [12], we consider a model in which agents face investment opportunities described by their cash flows. A filtered probability space $\left(\Omega, \mathcal{F},(\mathcal{F})_{t \in R_{+}}, \mathbf{P}\right)$ satisfying the usual conditions of saturatedness and right continuity is specified and fixed. The set $\Omega$ represents all possible states of the world.

We model investment opportunities which are available to investors in the following way. Let $X$ be as in Examples 4.3, $4.4 \mathrm{a}$ and $4.4 \mathrm{~b}$.

Definition 5.1. An investment is some $x \in X$.

Notice that from an economic point of view, this model is a generalization of the model considered in Jouini-Napp (2001) where $X=L^{1}(\widehat{\Omega}, \widehat{\mathcal{F}}, \widehat{\mu})$ as in Section 2 , as we now may also model in $X$ continuous time payment streams (which may or may not be absolutely continuous with respect to Lebesgue-measure).

We consider a convex cone $J \subseteq X$ of available investment opportunities. We are led to consider convex cones (instead of linear subspaces) in order to take into account the fact that investors are not necessarily able to sell an investment plan, as in the case of short sale constraints or transaction costs (see Jouini-Napp, 2001, for more details). We also suppose that $J$ contains $X_{-}$with the economic interpretation that agents are allowed to throw away money.

We now define arbitrage opportunities and free lunches. As in Examples 4.3, $4.4 \mathrm{a}$ and $4.4 \mathrm{~b}$ respectively, let $Y$ be the space of equivalence classes of adapted processes $y=\left(y_{t}\right)_{t \in R_{+}}$such that the trajectories $t \mapsto y_{t}(\omega)$ are uniformly bounded and càdlàg (resp. càdlàg or continuous), for almost all $\omega \in \Omega$, modulo the processes vanishing outside an evanescent set.

Definition 5.2. $\quad$ 1. An arbitrage opportunity for $J$ is an available investment $x \in J$, such that $x \in X_{+} \backslash\{0\}$.

2. A free lunch for $J$ is some $x \in X_{+} \backslash\{0\}$, such that there exists a net $\left(x_{\alpha}\right) \subset J$ converging to $x$. In other words a free lunch is an element $x \in X_{+} \backslash\{0\}$ lying in $\bar{J}^{\sigma(X, Y)}$, the closure of $J$ with respect to $\sigma(X, Y)$.

The definition of an arbitrage opportunity is clear enough and corresponds to the usual one. Since ${ }^{4} X_{+}=\left\{x:\langle x, y\rangle \geq 0 \forall y \in Y_{+}\right\}$, an arbitrage opportunity can equivalently be defined as some $x \in J$ such that $\langle x, y\rangle>0$ for all $y \in Y_{++}$.

\footnotetext{
${ }^{4}$ by a direct application of the monotone class theorem.
} 
The economic interpretation of this definition is the following (see Lakner (1993) for a similar interpretation). The set $Y_{++}$corresponds to all possible numéraire or discount processes. We suppose that each agent in the economy has a subjective numéraire process and each of these numéraire processes may be selected by an agent. In such a context, a subjective arbitrage opportunity for an agent with subjective numéraire process $y \in Y_{++}$is some $x \in J$, such that $\langle x, y\rangle>0$. Since we do not appropriate any special significance to an agent versus the rest of the agents, a (global) arbitrage opportunity comes from the consensus of all agents and we define an arbitrage opportunity as an investment opportunity which is considered as a subjective arbitrage opportunity by all possible agents. As for free lunches, a free lunch denoting the possibility of getting arbitrarily close to an arbitrage opportunity, we define a subjective free lunch for an agent with subjective numéraire process $y \in Y_{++}$as a net $\left(x_{\alpha}\right) \subset J$ and $x \in X$ such that $\langle x, y\rangle>0$, and $\left\langle x_{\alpha}-b_{\alpha}, y\right\rangle \rightarrow\langle x, y\rangle$ for $b_{\alpha} \geq 0$. Then a (global) free lunch is an investment opportunity, which is considered as a subjective free lunch by all possible agents.

Besides notice that as pointed out by Mas-Collel and Zame (1991), it is natural in economics to work with topologies compatible with the duality between a space of measures and a space of continuous functions. Essentially because with such topologies, cash streams at nearby times are considered as good substitutes. In our specific setting, if $t_{n}$ tends to $t$ (from above in the case of càdlàg, from below in the case of càglàd and in an arbitrary manner in the case of continuous trajectories), then, for any topology $\tau$ compatible with the duality $\langle X, Y\rangle$, the cash flow consisting of $\$ 1$ at date $t_{n}$ converges to the cash flow consisting of $\$ 1$ at date $t$ with respect to $\tau$. More generally, cash streams with approximately equal amounts at nearby times are "close". Therefore, if two cash streams are "close" in the usual sense, i.e., for the total variation, then they are close in our sense.

We are now in a position to state the following version of the Fundamental theorem of Asset Pricing.

Corollary 5.3. In the setting of Examples 4.3, $4.4 \mathrm{a}$ and $4.4 \mathrm{~b}$ the following conditions are equivalent:

1. There is no free lunch, i.e. $\bar{J}^{\sigma(X, Y)} \cap X_{+}=\{0\}$.

2. There is some $y \in Y_{++}$such that $\left.y\right|_{J} \leq 0$. 
This corollary enables to obtain a characterization of the no-free lunch condition by the existence of a càdlàg (resp. càglàd, resp. continuous) state price deflator or numéraire process, which restricted to $J$ is nonpositive. For the applications, the choice of the pair $(X, Y)$ and subsequently of the topology depends on the notion of closedness we want to adopt on the space of cash streams or on the properties we want on the numéraire processes.

\section{References}

[1] Ansel, J.P., Stricker, C. (1990) Quelques remarques sur un théorème de Yan. Sém. de probabilités. Lecture notes in Mathematics XXIV Springer-Verlag, New-York, 226-274.

[2] Clark, S. (1993) The valuation problem in arbitrage price theory. J. Math. Econ. 22, 46.

[3] Delbaen, F. (1992) Representing martingale measures when asset prices are continuous and bounded. Math. Fin. 2, p. 107-130.

[4] Delbaen, F., Schachermayer, W. (1994) A general version of the fundamental theorem of asset pricing. Math. Ann. 300, p. 463-520.

[5] Delbaen, F., Schachermayer, W. (1998) The fundamental theorem of asset pricing for unbounded stochastic processes. Math. Ann. 312, p. 215-250.

[6] Dellacherie, C. (1972) Capacités et processus stochastiques. Springer Verlag, Berlin.

[7] Dellacherie, C., Meyer, P.A. (1975-1980) Probabilités et potentiel. Hermann, Paris.

[8] Duffie, D., Huang, C. (1986) Multiperiod security markets with differential information: martingales and resolution times. J. Math. Econ., 15, p. 283303.

[9] Fremlin, D.H. Topological Riesz Spaces and Measure Theory. Cambridge University Press, 1974, ISBN 0-521-20170-5. 
[10] Frittelli, M., Lakner, P. (1994) Arbitrage and free lunch in a general financial market model; the fundamental theorem of asset pricing. In Mathematical Finance M.H.A. Davis, D. Duffie, W.H. Fleming and S.E. Shreve, Eds. Springer, NY.

[11] Halmos, P.R., Savage, L.J. (1949) Application of the Radon-Nikodym theorem to the theory of sufficient statistics. Ann. Math. Statist. 20, p. 225-241.

[12] Jouini, E., Napp, C. (2001) Arbitrage and investment opportunities. Fin. and Stoch., 5, 305-325.

[13] Kabanov, Yu.M., Kramkov, D.O. (1994) Large financial markets: asymptotic arbitrage and contiguity. Theory Probab. Appl. 39, p. 222-228.

[14] Kabanov, Yu.M., Kramkov, D.O. (1998) Asymptotic arbitrage in large financial markets. Finance and Stochastics, Vol. 2, issue 2.

[15] Klein, I., Schachermayer, W. (1996) Asymptotic Arbitrage in Non-Complete Large Financial Markets. Theory of Probability and its Applications, Vol. 41, No.4, 1996, p. 927-934.

[16] Klein, I., Schachermayer, W. (1996) A Quantitative and a Dual Version of the Halmos-Savage Theorem with Applications to Mathematical Finance. The Annals of Probability, Vol. 24, No. 2, p. 867-881.

[17] Kreps, D.M. (1981) Arbitrage and equilibrium in economies with infinitely many commodities. J. Math. Econ. 8, p. 15-35.

[18] Lakner, P. (1993) Martingale measures for a class of right-continuous processes. Math. Fin. 3(1), p. 43-53.

[19] Mas Colell, A., Zame, W. Equilibrium theory in infinite dimensional spaces in Handbook of Mathematical Economics (W. Hildenbrandt and H.Sonnenschein, eds.), vol.4., North Holland, Amsterdam.

[20] Schachermayer, W. (1994) Martingale measures for discrete time processes with infinite horizon. Math. Fin. 4, p. 25-55.

[21] Schachermayer, W. (2000) Some Remarks on a Paper of David Kreps. To appear in Journal of Mathematical Economics. 
[22] Semadeni, Z. Banach Spaces of Continuous Functions. Wroclawska Drukarnia Naukowa, Poland, 1971.

[23] Stricker, C. (1990) Arbitrage et lois de martingale. Ann. Inst. Henri Poincaré 26, p. 451-460.

[24] Yan, J.A. (1980) Caractérisation d'une classe d'ensembles convexes de $L^{1}$ ou $H^{1}$. Sém. de Probabilités XIV, Lect. Notes Mathematics 784, p. 220-222. 